\title{
Combined multiphoton fluorescence microscopy and photoacoustic imaging for stratigraphic analysis of paintings
}

\author{
George J. Tserevelakis, ${ }^{1, \dagger}$ (i) Vassilis Tsafas, ${ }^{1,2, \dagger}$ Kristalia Melessanaki, ${ }^{1}$ Giannis Zacharakis, ${ }^{1, *}$ and \\ GeORGE FILIPPIDIS ${ }^{1}$ \\ ${ }^{1}$ Foundation for Research and Technology Hellas, Institute of Electronic Structure and Laser, N. Plastira 100, Heraklion, Crete, Greece \\ ${ }^{2}$ Department of Physics, University of Crete, Heraklion, Crete, Greece \\ *Corresponding author: zahari@iesl.forth.gr
}

Received 7 November 2018; revised 17 December 2018; accepted 17 December 2018; posted 11 January 2019 (Doc. ID 351349); published 21 February 2019

\begin{abstract}
We demonstrate the effective combination of multiphoton and photoacoustic (PA) imaging for the high-resolution stratigraphic analysis of multilayered art objects with emphasis on paintings. A novel convolution-based algorithm is additionally applied for the precise discrimination of nonlinear signals, providing valuable information in regard to the thickness and composition of successive varnish and paint layers in the mock-up samples. On the other hand, PA contrast complements the extracted data by revealing well-hidden graphite underdrawings below the paint at high sensitivity levels. The final composite images are directly compared with cross-sectional brightfield observations, validating the capabilities of the bimodal diagnosis in terms of measurement accuracy and contrast specificity. The presented hybrid diagnostic approach has the potential to optimize delicate interventions in works of art such as the selective removal of aged materials, thus promoting a significantly improved restoration outcome. () 2019 Optical Society of America
\end{abstract}

https://doi.org/10.1364/OL.44.001154

Specialized diagnostics for works of art require in situ noninvasive methods, providing key information that is valuable for the promotion of optimized restoration interventions such as the selective removal of altered/aged materials. More specifically, for paintings, these delicate art conservation procedures can benefit from the prior knowledge of the artwork's surface topography, the specific determination of stratigraphic layers composed of different materials (e.g., varnishes, paints), and the precise structural mapping of the artwork, revealing possible cracks or detachments within its bulk. In this context, several optical or photoacoustic (PA) imaging techniques typically using near-infrared (NIR) radiation have been applied for the delineation of an artwork's complex internal structure.

During the last decade, optical coherence tomography has been successfully demonstrated to provide high-resolution cross-sectional images for the visualization of low scattering varnishes, as well as thin semi-transparent paint layers [1]. More recently, though, nonlinear microscopy approaches were introduced in regard to cultural heritage $(\mathrm{CH})$ diagnostics in an effort to extend the maximum imaging depth when looking into more scattering media, acquiring different kinds of contrast for assessing the composition of the used materials. In this direction, various studies have proven the potential of multiphoton excitation fluorescence (MPEF), second-harmonic generation and third-harmonic generation nonlinear contrast modes for depth-resolved imaging of fresh and aged varnishes $[2,3]$, lining glues [4], historical coatings [5], parchments [6], paints [7,8], and corrosion layers in metal artefacts [9]. Nevertheless, several materials typically met in works of art are optically opaque as a result of their inherently high scattering and absorption properties, not permitting observations beyond a few $\mu \mathrm{m}$ in depth using solely light. To overcome these issues, PA imaging has been very lately proposed as a highly promising diagnostic tool for $\mathrm{CH}$, which is able to provide optical absorption contrast through the detection of ultrasonic waves following excitation with pulsed laser radiation. Due to the up to three orders of magnitude lower attenuation coefficient of ultrasound compared to NIR radiation, imaging depths of several $\mathrm{mm}$ can be achieved in turbid media with a spatial resolution determined by acoustic detection parameters rather than by focused light [10]. Such novel PA imaging approaches have already been applied in $\mathrm{CH}$ for the visualization of hidden underdrawings in paintings [11], the thickness measurement of paint layers [12], as well as the uncovering of content in layered documents [13].

In this Letter, we have effectively combined MPEF microscopy with PA diagnosis through consecutive imaging sessions in order to provide precise stratigraphic information in works of art with emphasis on paintings. Despite the fact that the combination of these contrast modes has been exploited for biomedical imaging in previous studies [14], this is the first time, to the best of our knowledge, that such methodology is applied in $\mathrm{CH}$ diagnostics. The high contrast complementarity provided by 
this bimodal approach has additionally been enhanced by postprocessing of the acquired MPEF signals using a novel algorithm, which is able to discriminate overlapping layers according to the nonlinearity order and the optical parameters of the system. Finally, the combined images have been directly compared with cross-sectional brightfield observations, validating the capabilities of the proposed hybrid diagnostic method in terms of measurement accuracy and imaging specificity.

The nonlinear microscopy setup employed a single $200 \mathrm{fs}$ laser beam at $1028 \mathrm{~nm}$ (t-pulse, amplitude Systemes, Pessac, France; pulse repetition rate, $50 \mathrm{MHz}$ ) for the realization of MPEF imaging [Fig. 1]. During our studies, the damage threshold is avoided by using an average power less than $25 \mathrm{~mW}$ at the sample plane corresponding to pulse energy of $\sim 0.5 \mathrm{~nJ}$ [7]. A detailed description regarding the technical specifications of the reflection mode MPEF setup can be found elsewhere [7].

The PA imaging apparatus [Fig. 1] integrated a $Q$-switched Nd:YAG nanosecond laser source (QIR-1064-200-S, CrystaLaser LC, Reno, NV, U.S.; wavelength, $1064 \mathrm{~nm}$ ) for the efficient excitation of PA signals. The beam was loosely focused on the back side of the sample, and the generated PA waves were detected by a spherically focused immersion ultrasonic transducer. The experimental details of the PA imaging apparatus can be found in previous works [11]. A thin layer of $3 \% \mathrm{w} / \mathrm{v}$ carboxymethyl cellulose (CMC) gel was coated over the mock-up, serving as a coupling medium for the efficient propagation of the generated ultrasonic waves. The painting samples prepared for this Letter aimed to replicate real easel paintings on a small scale. Initially, patterns of parallel lines ( $\sim 0.5 \mathrm{~mm}$ thick) were produced on prepared canvas by employing a graphite pencil (CASTELL 9000 3B, Faber-Castell, Stein, Germany), representing the sketch layer of the painting. Subsequently, red lead pigment ( $\mathrm{Pb} 3 \mathrm{O} 4$, Minium, Kremer Pigments No. 42500) was mixed with an acrylic binder (Lascaux Acrylic Adhesive $498 \mathrm{HV}$ ) to produce an acrylic paint that was applied by brush over the sketch of the canvas. A thin layer of varnish (mastic or dammar) was finally applied on the paint to simulate the structure of realistic paintings.

An analysis and discrimination of the recorded MPEF was achieved through the development of a novel image processing

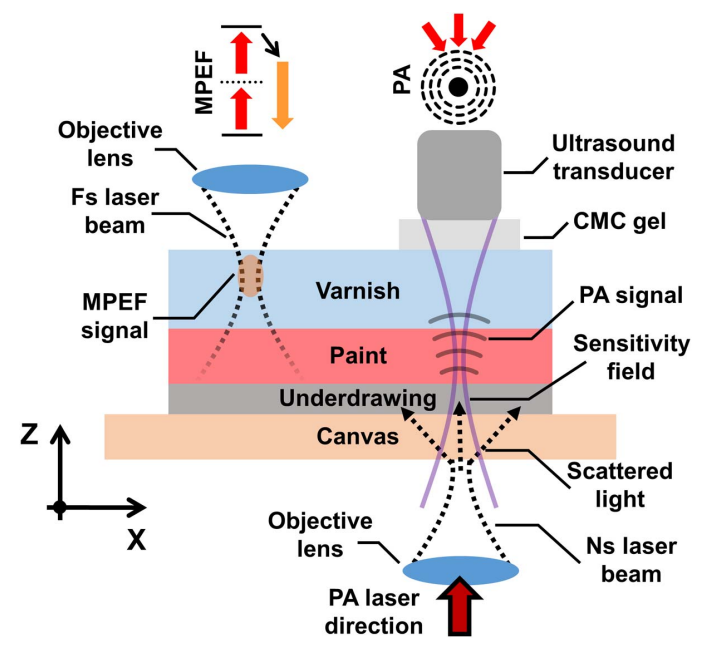

Fig. 1. Schematics of MPEF and PA experimental configurations for the cross-sectional imaging of a mock-up painting sample. algorithm in a MATLAB programming environment. The depth-resolved MPEF signal (along the Z-axis) for each lateral scanning position (the $\mathrm{X}$-axis) was considered to be the summation of two terms involving the nonlinear interaction of the focused Gaussian beam with the successive varnish and paint layers [Fig. 2(a)]. From a mathematical standpoint, the first term represents the convolution of the axial beam's profile raised to the third power, with a top-hat function corresponding to the overlying varnish layer. The exponentiation of the beam's profile is linked to the nonlinearity order based on the spectral characteristics of the materials. The investigated fresh varnishes present high transparency in the visible and infrared regions, while they absorb strongly in the near-UV part, thus demonstrating a predominant three-photon excitation mechanism for the employed excitation wavelength [15]. Similarly, the second term represents the convolution of the squared axial profile with a second top-hat function standing for the underlying paint layer, which presents strong twophoton absorption properties. The algorithm took additionally into account the scattering effects on the fundamental beam, as well as the partial re-absorption of the emitted fluorescence into the paint layer's bulk, by inserting an exponential attenuation factor in the second convolution term. The absorption effects in the varnish layer were considered to be negligible within the employed excitation and detection range, as has been shown in previous studies [3,7]. The developed mathematical model is used in an optimization fitting procedure for the determination of the first top-hat function's amplitude and width, which provides the varnish thickness and the relative depth of the varnish-paint interface. Since the scattering of the fundamental (a)

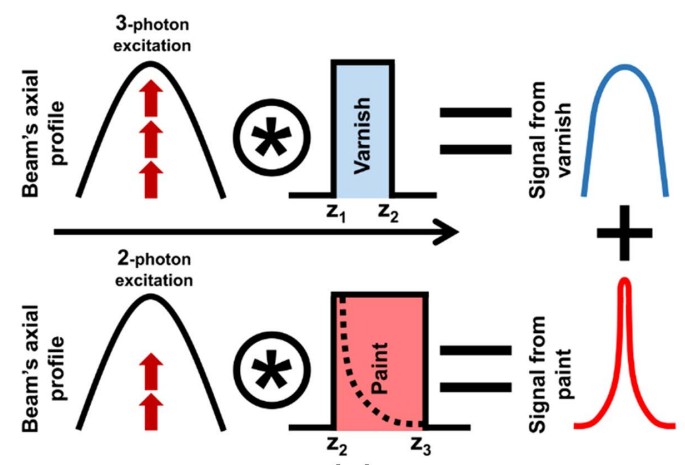

(b)

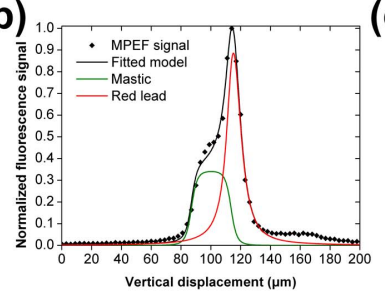

(c)

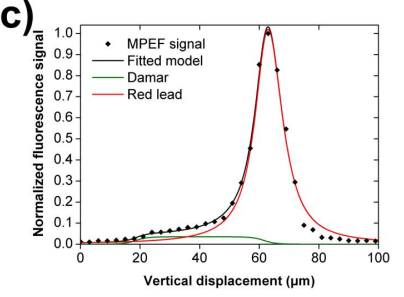

Fig. 2. MPEF signal discrimination in Z-Scan measurements. (a) Graphical illustration of the fitted model resulting from the summation of two convolution functions involving axial signal profiles and layer thicknesses, represented as top-hat functions. The optical attenuation has been additionally considered due to scattering and fluorescence re-absorption effects. (b) Z-Scan MPEF measurement of a red lead painting sample covered with mastic. The fitted model (black line, $\left.R^{2}=0.99\right)$ was used to decouple the recorded response (black points) into respective signal contributions arising from varnish (green line) and paint (red line). (c) Similar data are presented for a painting sample covered with dammar $\left(R^{2}=0.99\right)$. 
beam into the paint is very high, the second convolution term of the algorithm provided practically information about the maximum penetration depth, rather than the layer's thickness. The resulting depth values were finally corrected for apparent depth distortion due to the refractive index mismatch between air $(n=1)$ and measured layers $(n \approx 1.5)$.

The capability of the developed algorithm in regard to MPEF signal discrimination was initially evaluated by performing Z-Scan measurements in two painting samples covered with mastic and dammar varnishes, respectively [Figs. 2(b) and 2(c)]. Black points correspond to the recorded MPEF signal values as a function of depth, whereas black curve represents the optimum fitting of experimental data according to the employed convolution model $\left(R^{2}=0.99\right.$ for both cases), which can be subsequently decoupled into distinct contributions arising from each layer. More specifically, the green curve stands for the first convolution term, representing the interaction of excitation beam with the varnish, whereas the red curve describes the second term indicating the penetration depth of the fundamental beam into the paint's bulk. In the case of mastic sample [Fig. 2(b)], the varnish boundaries were estimated at $z_{1}=87 \mu \mathrm{m}$ and $z_{2}=114 \mu \mathrm{m}$, respectively, yielding an uncorrected thickness equal to $27 \mu \mathrm{m}$. By multiplying the latter value with the apparent depth correction factor $(\sim 1.6$ for the employed objective lens) [16], a final varnish thickness of $43 \mu \mathrm{m}$ is calculated. On the other hand, the $1 / e$ width of the red curve was estimated at $15 \mu \mathrm{m}$, yielding a penetration of around $24 \mu \mathrm{m}$ into the paint.

Similarly, the dammar sample's varnish boundary values were calculated at $z_{1}=19 \mu \mathrm{m}$ and $z_{2}=62 \mu \mathrm{m}$, providing a final distortion-corrected thickness of $\sim 69 \mu \mathrm{m}$ [Fig. 2(c)]. As expected, the penetration depth into the paint was found to be identical to the previous case. It is also worth mentioning that the amplitudes of the mastic and dammar signal contributions present a significant difference by almost an order of magnitude, thus allowing for their reliable identification through the intensity-based analysis of the recorded MPEF. The increased absorption coefficient of mastic at $343 \mathrm{~nm}$ (threephoton excitation in our case) can be linked with the higher MPEF intensity signals recorded from this material.

Regarding the PA imaging session, we initially recorded signals arising from several hidden graphite regions of the mastic mock-up sample in order to adjust the system's parameters for efficient detection of underdrawings. A characteristic PA signal is shown in Fig. 3(a) (black line), whereas the respective red curve represents the modulus of the signal's Hilbert transformation, which is typically used for the generation of cross-sectional images or $3 \mathrm{D}$ reconstructions. The delayed low amplitude peaks following the main signal can be attributed to multiple reflections of PA waves; therefore, they were safely excluded from subsequent analysis. Figure 3(b) shows a brightfield image of the investigated pencil sketched canvas prior the application of red lead paint and mastic layers, whereas Fig. 3(c) corresponds to the final mock-up painting sample. A 200 by 200 pixels maximum amplitude projection (MAP) PA image for the central $1 \times 1 \mathrm{~cm}^{2}$ region [white square in Fig. 3(c)] is shown in Fig. 3(d), revealing clearly the underlying pencil lines at high contrast, as a result of the selective absorption of NIR radiation by graphite.

Having evaluated the distinct capabilities of the employed stand-alone systems, we finally proceeded to the combined
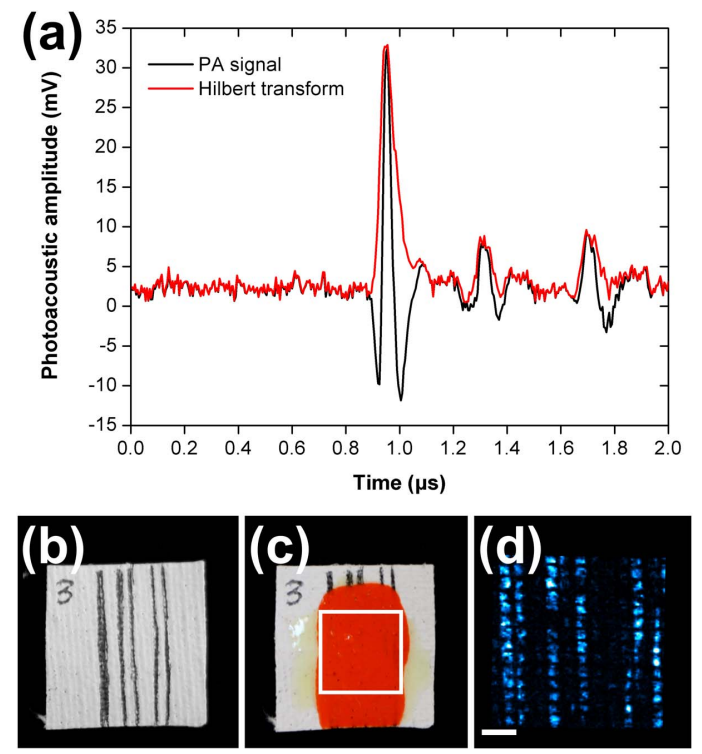

Fig. 3. PA imaging of underdrawings. (a) Characteristic timedomain PA signal arising from graphite regions on a mastic covered painting sample (black line) and the respective modulus of its Hilbert transformation (red line). (b) Brightfield image of a mock-up canvas sample $\left(22 \times 22 \mathrm{~mm}^{2}\right)$ with several sketch lines drawn by pencil. (c) Same mock-up following the application of red lead paint and mastic layers. The white square $\left(1 \times 1 \mathrm{~cm}^{2}\right)$ indicates the central region imaged using the PA modality. (d) MAP PA image of underlying pencil lines for the selected field of view (scale bar, $2 \mathrm{~mm}$ ).

MPEF and PA imaging of the mastic mock-up sample following two sequential sessions. Figure 4(a) shows the sample's central cross-sectional MPEF image (perpendicular to sketch lines) for an XZ region of $1 \mathrm{~cm}$ by $385 \mu \mathrm{m}$ sampled with 200 by 80 pixels, respectively. The small regions characterized by a total absence of MPEF signals can be attributed to the high inhomogeneity of the handmade painting sample. By applying the developed algorithm, the mixed information carried by the MPEF image was decomposed into relative contributions from the mastic layer [Fig. 4(b)] and red lead paint [Fig. 4(c)] presented with green and red pseudo colors, respectively. The cross-sectional PA image from the same region shown in Fig. 4(d) reveals explicitly the distribution of graphite along the selected central line. The apparent low axial resolution can be attributed to the finite bandwidth of the ultrasonic transducer, as well as the drastic degradation of high acoustic frequency components during their propagation through the overlying layers. A composite image merging the sections presented in Figs. 4(b)-4(d) is shown in Fig. 4(e), delineating the full stratigraphy of the investigated mock-up sample at high resolution and contrast specificity within the limits of the combined imaging approaches. The superposition of the profiles in the $\mathrm{Z}$-axis has been achieved according to the mean thickness of the mock-up at the measurement region, as has been estimated by brightfield microscopy observations at the edges of the sample.

Finally, to validate our results, we cut the mock-up sample at the imaged profile's location and obtained cross-sectional brightfield microscopy images to use them for a direct comparison with the composite image. Figure 4(f) shows a near-UV brightfield view of the sample's cross section at the region 

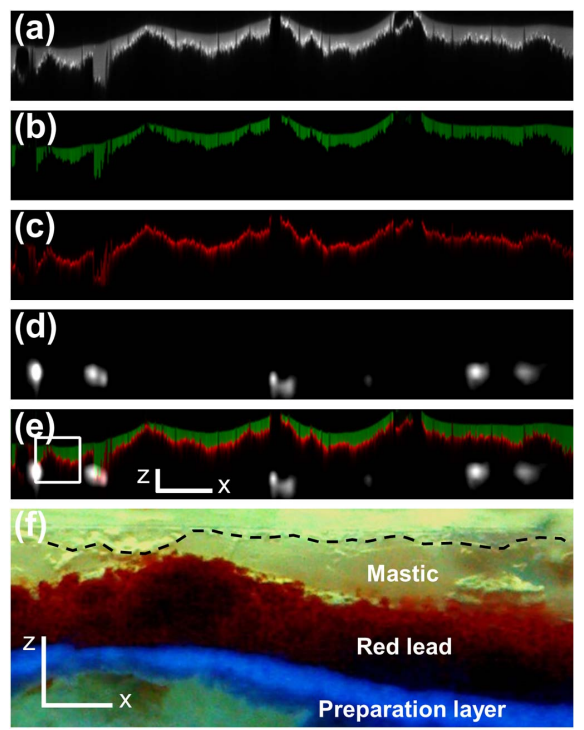

Fig. 4. Combined MPEF and PA cross-sectional imaging. (a) Raw MPEF image. (b) Unmixed MPEF image of the mastic layer. (c) Unmixed MPEF image of the red lead paint layer. (d) PA image of the underlying pencil sketch. (e) Composite MPEF and PA image (scale bar Z, $100 \mu \mathrm{m}$; scale bar X, $1 \mathrm{~mm}$ ). (f) Brightfield image of the region indicated with a white square in (e) using near-UV light. The black dashed line represents the border of the mastic layer. Both scale bars in the $\mathrm{Z}$ and $\mathrm{X}$ dimensions correspond to $100 \mu \mathrm{m}$.

indicated with a white square in Fig. 4(e). As is clear from the images, the two regions present an obvious similarity in terms of structure, morphology, and layer spatial distribution on the canvas. Furthermore, from a quantitative point of view, the extracted mastic layer thickness value for the right section of the region of interest $(\sim 75 \mu \mathrm{m})$ is in very good agreement with Fig. 4(f), thus confirming the precision of our measurements following convolution-based data processing.

During the previous years, multiphoton microscopy has been demonstrated as a non-invasive, high- resolution diagnostic tool for $\mathrm{CH}$ applications. In this Letter, we developed a novel model-based MPEF signal analysis algorithm to provide insights regarding the identification of two different varnish layers, as well as the precise discrimination and measurement of successive varnish and paint layers in artworks. The estimated attenuation coefficient through the paint layer could be potentially used for the investigation of unknown pigment types. Furthermore, the detection of MPEF signals in the reflection mode and the wide scanning regions $(\sim \mathrm{cm})$ offer the capability of evaluating original art objects. Nevertheless, multiphoton modalities present a relatively limited penetration in scattering media and, for this reason, PA imaging was additionally applied for the visualization of hidden underdrawings in the investigated mock-up painting samples with high sensitivity and contrast. In this Letter, a 3\% w/v CMC gel layer has been used as a highly compatible immersion medium [11] for the efficient detection of the generated PA signals. Nevertheless, in the case of sensitive paint surfaces that could not tolerate contact with any gel material, the option of immersion medium-free PA imaging could be considered by incorporating a fully non-contact interferometric ultrasonic detector, or highefficiency air-coupled transducers [13]. This Letter has provided experimental evidence that the effective combination of these two diagnostic techniques can offer highly complementary information, allowing for the accurate mapping and delineation of different stratigraphic layers in a painted artwork. A compact imaging apparatus integrating multiphoton and PA contrast modes in a single instrument constitutes our main future target. It is anticipated that such a combined diagnostic approach will be valuable for the art conservation community, finding applications in real case studies of several $\mathrm{CH}$ objects.

Funding. IPERION CH-H2020 (654028); POLITEIA II (MIS 5002478); Stavros Niarchos Foundation (ARCHERS); General Secretariat for Research and Technology and Hellenic Foundation for Research and Innovation (GSRT \& HFRRI); HELLAS CH (MIS 5002735).

†These authors contributed equally to this Letter.

\section{REFERENCES}

1. C. S. Cheung, J. M. O. Daniel, M. Tokurakawa, W. A. Clarkson, and H. Liang, Opt. Lett. 39, 6509 (2014).

2. G. Filippidis, M. Mari, L. Kelegkouri, A. Philippidis, A. Selimis, K. Melessanaki, M. Sygletou, and C. Fotakis, Microsc. Microanal. 21, 510 (2015).

3. M. Oujja, S. Psilodimitrakopoulos, E. Carrasco, M. Sanz, A. Philippidis, A. Selimis, P. Pouli, G. Filippidis, and M. Castillejo, Phys. Chem. Chem. Phys. 19, 22836 (2017).

4. S. Psilodimitrakopoulos, E. Gavgiotaki, K. Melessanaki, V. Tsafas, and G. Filippidis, Microsc. Microanal. 22, 1072 (2016).

5. G. Latour, J.-P. Echard, M. Didier, and M.-C. Schanne-Klein, Opt. Express 20, 24623 (2012).

6. G. Latour, L. Robinet, A. Dazzi, F. Portier, A. Deniset-Besseau, and M.-C. Schanne-Klein, Sci. Rep. 6, 26344 (2016).

7. G. Filippidis, G. J. Tserevelakis, A. Selimis, and C. Fotakis, Appl. Phys. A 118, 417 (2015).

8. A. Dal Fovo, M. Oujja, M. Sanz, A. Martínez-Hernández, M. V. Cañamares, M. Castillejo, and R. Fontana, Spectrochim. Acta A 208, 262 (2019).

9. F. Faraldi, G. J. Tserevelakis, G. Filippidis, G. M. Ingo, C. Riccucci, and C. Fotakis, Appl. Phys. A 111, 177 (2013).

10. J. Yao and L. V. Wang, Laser Photonics Rev. 7, 758 (2013).

11. G. J. Tserevelakis, I. Vrouvaki, P. Siozos, K. Melessanaki, K. Hatzigiannakis, C. Fotakis, and G. Zacharakis, Sci. Rep. 7, 747 (2017).

12. G. J. Tserevelakis, A. Dal Fovo, K. Melessanaki, R. Fontana, and G. Zacharakis, J. Appl. Phys. 123, 123102 (2018).

13. G. J. Tserevelakis, M. Tsagkaraki, P. Siozos, and G. Zacharakis, Strain 54, e12289 (2018).

14. M. Seeger, A. Karlas, D. Soliman, J. Pelisek, and V. Ntziachristos, Photoacoustics 4, 102 (2016).

15. G. Filippidis, E. J. Gualda, K. Melessanaki, and C. Fotakis, Opt. Lett. 33, 240 (2008).

16. A. Selimis, G. J. Tserevelakis, S. Kogou, P. Pouli, G. Filippidis, N. Sapogova, N. Bityurin, and C. Fotakis, Opt. Express 20, 3990 (2012). 